\title{
A Cost Estimation Tool Integrated into FIPER
}

\author{
David Koonce, Robert Judd, Thomas Keyser \\ Ohio University, Athens OH, 45701 USA \\ Em: koonce@ohio.edu.
}

Keywords FIPER, Cost Estimation,

\begin{abstract}
The estimation of a part's manufacturing cost in all phases of the design stage is crucial to concurrent engineering. To better estimate the cost for a product, data must be available from both engineering systems and business systems. This paper presents a cost estimation system being developed to support design time cost estimation using FIPER; the Federated Intelligent Product Environment, which is being developed as part of the NIST Advanced Technology Program. The FIPER project will be developing an architecture that interconnects design and analysis software tools in a peer level architecture using JAVA to support Multidisciplinary Design Optimisation, Design for Six Sigma and Robust Design.
\end{abstract}

\section{INTRODUCTION}

One key constraint in engineering design is product cost. However, estimation of the cost of a yet to be produced part or product is a difficult process. The NIST ATP sponsored FIPER project allows for a new and truly integrated cost estimation process for aiding design time cost analysis. FIPER will unify design tools for optimisation across multiple analytical disciplines. For cost to be included in this optimisation, a new and highly integrated cost estimation tool must be available. This tool will include the capability of developing cost estimates of a product from whatever data is available in the environment. In the early stages, simple parametric cost relations, like weight-based relations may be used. As more information is developed, cost estimates will be developed on design features, manufacturing features or even a developed process plan will automatically be substituted for the parametric relations. All estimates will also be adjusted for bias using a scaling factor determined by comparing the estimated cost of a similar existing part to the known actual cost of that part. 
This paper presents the design for a new highly customisable cost integration tool to support design time optimisation that considers cost as a constraint.

\section{FIPER}

FIPER, an acronym for Federated Intelligent Production EnviRonment, will utilize a technology called Intelligent Master Modelling (IMM) to allow design engineers to reduce the time to evaluate potential designs across all analytical disciplines. FIPER will support advanced design methodologies such as DFSS (Design For Six Sigma), MDO (Multidisciplinary Design Optimisation) and robust design. While the IMM helps coordinate design and analysis, a supporting architecture will be built upon the concepts of federated information systems. The FIPER infrastructure is being developed entirely in JAVA to support the mixed computing platforms typical in product design.

FIPER is being developed as part of a four-year National Institute of Standards and Technology (NIST) Advanced Technology Program (ATP). The development team consists of the General Electric Corporation, BFGoodrich Aerospace Aerostructures Group, Engineous Software, Parker Hannifin Corporation, Ohio University, Stanford University and the Ohio Aerospace Institute. In specifying the capabilities and services in FIPER, the development team addressed five problems common to many businesses in the United States:

- the need to reduce time to market,

- the need to reduce design cycle time,

- the need to reduce product costs,

- the need to improve product performance, and

- the need to improve product quality and reliability.

As a consequence of the need to reduce production costs, FIPER must have the ability to accurately predict the cost of a potential design. The software tool that produces the estimates must operate in the FIPER environment and be able to, with no user interaction, generate a cost estimate as a service to a calling program.

\section{MANUFACTURING COST ESTIMATION}

It is often posited that the major portion of a product's cost, as much as $80 \%$, is determined early in the design process. Decisions like material can easily be seen to impact cost. However, decisions like a radius or blend may result in the need for a tool change, new setup or even a processing machine 
change, adding to the manufacturing cost of a part. As such, producibility often figures into the estimation of a part's cost. Regardless, functional specifications usually drive the design process [1].

Despite the importance of the design details to product cost, a recent study found the delay between the design decision and cost determination hindered the designer's ability to learn about the process implications of design decisions [2]. In addition, the consequences of the costly decisions were often not fed back to designers at all [2].

Numerous commercial cost estimation tools exist and many organizations have developed proprietary cost estimation systems. The sophistication of these tools ranges from spreadsheets to multi-user mainframe database systems. The capability of these systems ranges from the ability to estimate costs for highly specific parts to generic systems which can be used to estimate costs for virtually any manufactured part. Regardless of the sophistication or size of the system, the manufacturing cost of a part is estimated using one or more of four basic methods: intuitive, analogous, parametric and analytical [3].

The intuitive approach relies on the experience of the estimator to predict the cost. An analogical estimate is essentially a variant estimate using similar parts, often matched using a group technology code. Parametric estimates use the values of key part attributes to determine the cost. A parametric estimate may rely on very high-level parameters of the product's performance or use detailed geometric data. Lastly, an analytical estimate relies on a summation of the steps in the production process; and as such, can only be done late in the design process.

A newer trend in cost estimation is the inclusion of manufacturing system costs in the estimation of a part's cost. Various methods in this area include: activity based costing, throughput accounting, target costing, lifecycle costing and strategic accounting [4]. For example, traditional activity based costing (ABC) can be further decomposed into activity based costs such as processing cost and non-activity based costs such as inventory holding costs [5].

Costs such as these are usually buried in an overhead factor. Overhead is also an area of cost estimation research with the notion that product complexity is the primary driver for overhead [6]. This study indicated that production volume and the number of transactions like engineering change orders strongly correlated with manufacturing overhead costs. For example, production volume may necessitate a need for increasing capacity or even process capability in the manufacturing system. 


\section{COST TOOL ARCHITECTURE}

The architecture of the FIPER cost estimation tool (FCET) is largely influenced by the cost estimation methodologies employed. FCET will use a combination of generative and variant costing, with designs being evaluated using either a work breakdown structure or a parameter based estimation from a similar part. This dual estimation approach will require that the tool maintain a repository of existing parts, with costs and match parameters, as well as traditional cost estimation equations and associated data files.

The FCET will consist of three separate user interfaces, a cost estimation engine and an associated database for storing estimates, and operational data. The three interfaces are the module builder, the template builder and the cost estimator. The end user interface, the cost estimator, will allow the estimation of parts using predefined structures to produce high-fidelity estimates. Figure 1 shows a simplified architecture on the tool.

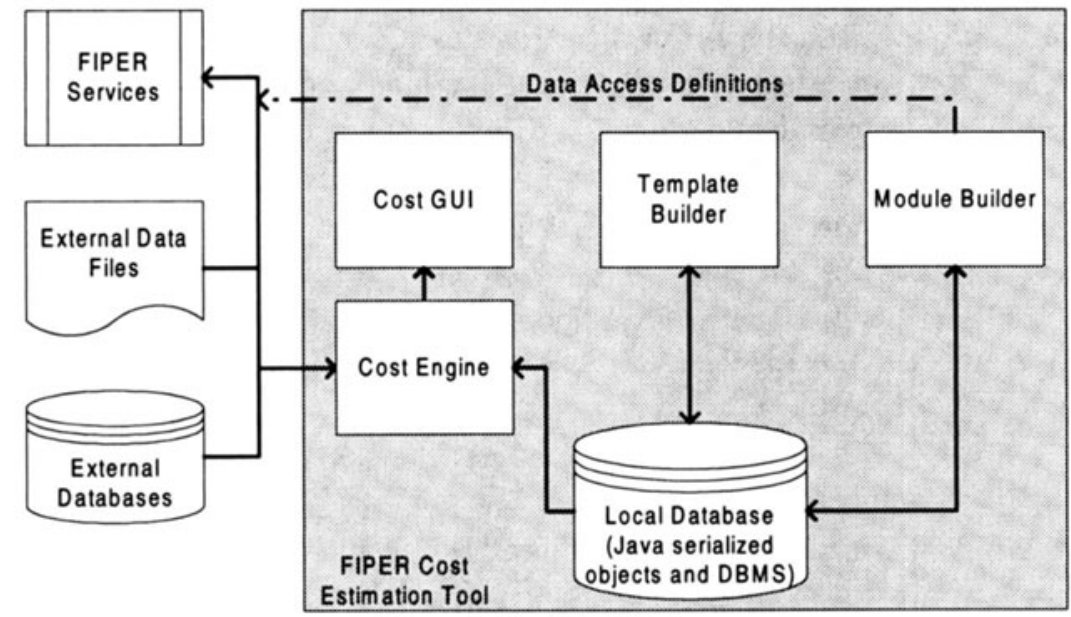

Figure 1. Tool Architecture

\section{The Development Environment}

The developer is presented with two tools: the module builder and the template builder. Dividing the development environment allows the separation between how costs are estimated from the view that the user is presented. This is analogous to database design, where the design of the schema is separate from the design of the end user data access forms. This allows for different views of the estimate, based on user needs. (e.g. an engineer may need a different view than a manager) 


\section{User Environment}

The user is presented with a tool that can call up any template. The costs in the template are divided into elements with focus on a work breakdown structure. For detailed designs, a work breakdown structure (WBS) can be utilized to generate detailed cost estimates. The WBS is a hierarchy consisting of three classes of modules: operations, components and assemblies. The WBS is generated for a specific estimate with pre-defined template.

Associated with each element is a group of attributes that are set by the user. These attributes may also be a default value or take their value from an element higher in the WBS. The template specifies a default value or relationships. For example, a high-level element may specify a material attribute, which is used for the material attribute in all machining sub-elements.

\section{Modules}

Modules form the basis for all estimates. They contain the definitions of the variables necessary for the cost equations and the definitions to the linkages to external data sources. Stored as executable JAVA objects, modules are usable in many cost estimates and are the instantiation of an organization's product families and processing logic.

Modules can use any cost method: parametric, feature based, operation based, $\mathrm{ABC}$, etc. The module builder allows the developer to create modules in a high-level GUI environment, which does not require programming experience.

\section{Templates}

A template is a group of elements, which can be used to estimate the cost of a product. At the simplest level, a template is a set of wrapped modules that are linked with defined relationships, defaults and mappings that enable the costs to be rolled-up into a compete cost estimate. Elements can be configured with:

- a set of input attributes (with defaults),

- a set of buckets (for summing calculated values),

- a set of fixed sub-elements,

- a set of optional sub-elements,

- a set of modules,

- mappings between element variables and sub-element variables, and

- matching rules for scaling an estimate. 
Templates allow for interaction between the variables and equations in the modules that make up a cost estimate. For example, a material variable in one element (like a machining operation module) may be linked to the material of a higher-level element. The linkage then allows a material change to cascade through the cost estimate.

Templates are not generated at the time of estimation. They are a library of products and processing steps predefined by cost estimation experts. While a basic set of process templates may be applicable to many organizations, it will be necessary that the FCET be customized for each implementation.

Templates can also be used to present the user with a view different from than what is provided in the constituent modules. For example, the element attributes may present a geometric feature with the element attributes while the modules are operation based. All necessary mappings and translations are maintained in the template structure.

\section{Risk Analysis}

The cost estimation tool will have the ability to perform risk analysis. The risk analysis will be done using Monte Carlo simulation. Given a set of inputs that may be any combination of deterministic and probabilistic values the Monte Carlo simulation will generate minimum, maximum and average cost estimates. Additionally, the variance will be reported as well as a graphical representation of the resultant distribution. The cost estimates will be based on user defined confidence interval levels (e.g. $90 \%, 95 \% \ldots$ ) or a user defined run length (number of simulated trials).

\section{Scaling}

Key to cost estimation is the minimization of error in the equations estimating the cost. One method for minimizing error is to use cost estimates of existing parts. By comparing computed cost with actual cost, a measure of the error can be determined and applied to a new estimate for a similar product. Figure 3 shows the equation for scaling a cost estimate using a closely matched part. The keys to scaling are one, having a complete and accurate database of current part costs and two, determining which existing part most closely matched the planned part.

$$
\begin{aligned}
& C_{S}=C_{U} \frac{M_{A}}{M_{E}} \\
& \text { Where, } \\
& C_{S} \text { Scaled cost estimate } \\
& C_{U} \text { Estimated cost of the part } \\
& \mathrm{M}_{\mathrm{A}} \text { Actual cost of matched part } \\
& \mathrm{M}_{\mathrm{E}} \text { Estimated cost of matched part }
\end{aligned}
$$

Figure 2. Cost Scaling Equation 
While the calculation for scaling is not complicated, determining a closely matching part may be difficult. A set of matching parameters must be defined for each element. For scaling to work, the error in the equations must be duplicated with both sets of calculations. For example, if two parts with very similar parameters use significantly different materials, then the method for estimating the costs may be entirely different. In this case, scaling the new part against the old part could add only random error. Beyond scaling processing cost, labour scaling is possible if data is available. However, for purchased parts, material and labour are combined which makes decomposition for scaling more difficult.

\section{Trade Studies}

The cost estimation tool will also store and track trade studies. Engineers will be able to set a reference point and then perform what-if analysis using multiple scenarios. At any time the user may reset the cost of any element or sub-element to a stored value in the trade study.

\section{CONCLUSIONS}

This paper has presented an architecture for a new cost estimation tool capable of generating estimates at all stages of the design process. FIPER presents an excellent opportunity for the creation of a design time cost estimation tool. With FIPER providing the necessary design data and a directed search toward optimality with respect to cost and other constraints, the FIPER cost estimation tool will help designers reduce costs while meeting performance constraints.

\section{REFERENCES}

[1] Locascio, A. (2000). Manufacturing Cost Modelling for Product Design, Journal of Flexible Manufacturing Systems, 12, 207-217

[2] Busby, J.S. (1997). The Limited Informativeness of Resource Discrepancy Feedback to Designers, Journal of Operations and Production Management, 17, 6, 630-646

[3] Duverlie, P. and Castelain, P. (1999). Cost Estimation During Design Step: Parametric Method versus Case Based Reasoning Method, Journal of Advanced Manufacturing Technology, 15, 895-906

[4] Boons, A. (1998), Product Costing for Complex Manufacturing Systems, Journal of Production Economics, 55, 241-255

[5] Park, C. and Kim, G. (1995). An Economic Model for Advanced Manufacturing Systems Using Activity Based Costing, Journal of Manufacturing Systems, 14, 6.

[6] Banker, R.D., Potter, G., and Schroeder, R.G. (1995). An Empirical Analysis of Manufacturing Overhead Cost Drivers, Journal of Accounting and Economics, 19, 115137 